\title{
J oint Space-time Coding and Power Domain Non-orthogonal Multiple Access for Future Wireless System
}

\author{
Jin Xu*", Hanqing Ding, Zeqi Yu, Zhe Zhang, Weihua Liu and Xueyan Chen \\ School of Information and Communication Engineering, \\ Zhengzhou University of Light Industry, Zheng Zhou, China 450001 \\ [e-mail:buptxujin@163.com, dinghanqing@zzuli.edu.cn] \\ *Corresponding author: Jin Xu
}

Received April 9, 2019; revised July 15, 2019; accepted September 15, 2019;

published January 31, 2020

\begin{abstract}
According to information theory, non-orthogonal transmission can achieve the multiple-user channel capacity with an onion-peeling like successive interference cancellation (SIC) based detection followed by a capacity approaching channel code. However, in multiple antenna system, due to the unideal characteristic of the SIC detector, the residual interference propagated to the next detection stage will significantly degrade the detection performance of spatial data layers. To overcome this problem, we proposed a modified power-domain non-orthogonal multiple access (P-NOMA) scheme joint designed with space-time coding for multiple input multiple output (MIMO) NOMA system. First, with proper power allocation for each user, inter-user signals can be separated from each other for NOMA detection. Second, a well-designed quasi-orthogonal space-time block code (QO-STBC) was employed to facilitate the SIC-based MIMO detection of spatial data layers within each user. Last, we proposed an optimization algorithm to assign channel coding rates to balance the bit error rate (BER) performance of those spatial data layers for each user. Link-level performance simulation results demonstrate that the proposed time-space-power domain joint transmission scheme performs better than the traditional P-NOMA scheme. Furthermore, the proposed algorithm is of low complexity and easy to implement.
\end{abstract}

Keywords: NOMA, P-NOMA, SIC, QO-STBC, coding rate optimization

A preliminary version of this paper appeared in 9th International Conference on Communications and Networking in China, Maoming, pp. 266-270, Guangdong, China. This version includes a concrete analysis and supporting implementation results on P-NOMA. 


\section{Introduction}

In current, orthogonal multiple aceess (OMA) such as orthogonal freqency division multplexing access (OFDMA) is widely used in long term evolutiion (LTE) system. In OMA system, to achieve a sufficiently high system throughput, we need to allocate the signal dimensions in terms of time or frequency to the near user which has good channel conditions. In this way, near user can approach its capacity and dominate the system throughput. However, the cell-edge user is not seved at all which is unfair to cell-edge user [1]. On the other hand, if we assign more signal dimensions to cell-edge user and less to near user, then the near user performs poor which thus leads to a low system throughput. As a result, we need to realize a trade-off between system throughput and user fairness.

In future mobile communication system, both spectral efficiency and user fairness are becoming more and more important such as in internet of things (IoT) applications. For this pupose, it is shown better to employ non-orthogonal multiple access (NOMA) scheme which schedules two or more user to the same resource [1],[2]. As shown in [3], NOMA combined with successive interference cancellation (SIC) can achieve the capacity region of single-input single-output (SISO) multiuser system in information theroy perspective. The cost we must pay is the higher compuational complexity since inter-user interference must be tackled in NOMA system. Several practical NOMA schemes, such as power-domain non-orthogonal multiple access (P-NOMA) [4], sparse code multiple access (SCMA) [5], pattern division multiple access (PDMA) [6], multi-user shared access (MUSA) [7] and interleaved division multiple access (IDMA) [8], etc, attract great attentions in fifth-generation (5G) mobile communication standard [9]. Roughly speaking, the last four NOMA schemes are based on code-domain division multiplexing while P-NOMA is power-domain division multiplexing based one. All these NOMA schemes by squeezing more users over limited time-frequence resource and employing optimal/near-optimal multi-user detection algorithm can improve both the throughput of cell-edge user and system throughput over theirs OMA based counterparts. However, in multiple input multiple output (MIMO) multiuser system, this is much more complicate. As shown in [10], if the transceiver has the full channel state information (CSI) knownledge, dirty paper coding (DPC) with SIC can achieve the MIMO broadcast channel (MIMO-BC) capacity. Unfortunately, for cellular communication system, full CSI feedback is costly and impracticable. In [11], a downlink P-NOMA scheme using intra-beam superposition coding and SIC is proposed for MIMO downlink multiuser system, SIC is carried out on the spatially-filtered scalar received signal to remove the inter-user interference within a beam due to inter-beam superpostion coding. Compare with DPC scheme, the proposed scheme in [10] is much easy to implement since the SIC is rely on the CSI at the receiver side. In [12], cooperative NOMA is proprosed to enhance the performance of the cell-edge user. In [13-15], peformance of cooperative NOMA network with hardware impairment and channel estimation error is studied.

The performance of interference cancellation (IC) is crucial to NOMA system since perfect IC is the premise of the acquisition of multiple access capacity. However, in practice, due to the imperfect performance of the SIC detector, the residual interference propagated to the next detection stage will significantly degrade the receiver performance [16]. To overcome this problem, in this work we proposed an improved P-NOMA scheme combined with 
space-time coding which is SIC-amenable for MIMO downlink system. First, take into account the user fairness, we employ fairness as a power allocation criterion. Under this condition, with proper power allocation inter-user streams can be separated from each other with SIC-based detector for P-NOMA. Second, a well-designed quasi-orthogonal space-time code (QO-STBC) was employed to facilitate the SIC-based MIMO detection of spatial data layers within each user. Third, to further improve the overall performance, we introduce multilevel-coding into QO-STBC coded P-NOMA which leads to the multiple-layer multiple-rate (MLMR) coded P-NOMA scheme. At last, We proposed an optimization algorithm to assign different channel coding rates to balance the bit error rate (BER) performance of different data layers for each user. Link-level performance simulation results demonstrate that the proposed three dimensional joint transmission scheme performs better than the traditional P-NOMA scheme. Furthermore, the proposed algorithm is of low complexity and easy to implement.

The rest of this paper is organized as follows. Section 2 is a review of P-NOMA system model and SIC detectin algorithm for SISO system. Section 3 is about the QO-STBC pattern matrix, the corresponding two-stage SIC detection algorithm and coding rate optimization algorithm for the proposed MLMR-coded P-NOMA. Section 4 is about the BER performance and link-level throughput simulation results. Section 5 is the conclusion of this paper.

\section{Review of P-NOMA system}

\subsection{System model of P-NOMA}

First, we consider a downlink multi-user system in which the base station (BS) scheduled two users on the same time-frequency resources. For simplicity, we first assume that the BS equipped with only a single antenna, its extension to multiple antennas will be described in the next section in detail. As shown in Fig. 1, user 1 is a cell-edge user (far user) with instantaneous signal-to-noise ratio (SNR), denoted by $S N R_{1}$, and user 2 is a near user with instantaneous SNR, denoted by $S N R_{2}$. Let $s_{1}$ and $s_{2}$ denote the signals intend to user 1 and user 2 respectively. After proper power allocation, the base-station transmits the combined signal, denoted as s, with nominalized power, to both users, where $E\left[\mid s^{2}\right]=\mathbf{1}$. We have

$$
s=\sqrt{1-\alpha s_{1}}+\sqrt{\alpha} s_{2}
$$

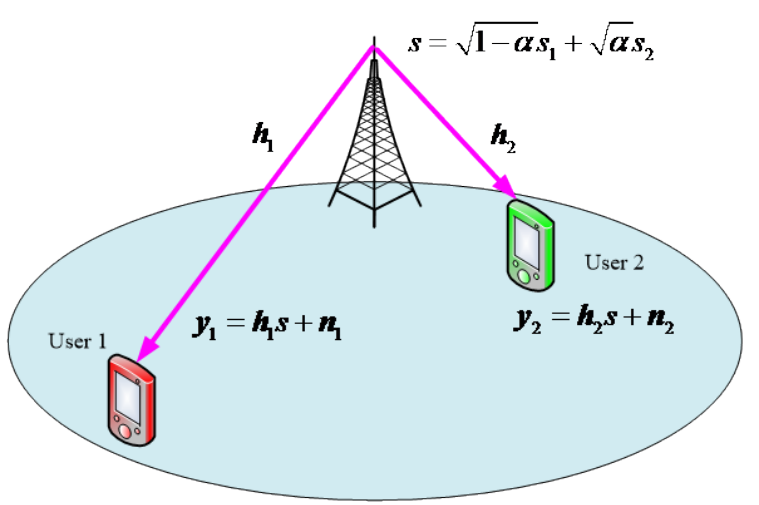

Fig. 1. System model of downlink P-NOMA 
where $\alpha$ denotes the power distribution factor associated to user 2 . Assuming that the channel coefficient vectors from the BS to user 1 and user 2 are $\boldsymbol{h}_{1}$ and $\boldsymbol{h}_{2}$ respectively, where $E\left[\left|\boldsymbol{h}_{1}\right|^{2}\right]=1$ and $E\left[\left|\boldsymbol{h}_{2}\right|^{2}\right]=1$. At the receiver side, the received discrete baseband signal of user 1 can be written as

$$
\boldsymbol{y}_{1}=\boldsymbol{h}_{1}\left(\sqrt{1-\alpha} s_{1}+\sqrt{\alpha} s_{2}\right)+\boldsymbol{n}_{1}
$$

where $\boldsymbol{n}_{1}$ is the additive white Gaussian noise (AWGN) and $\boldsymbol{n}_{1} \sim C N\left(0, \sigma_{1}^{2} \boldsymbol{I}\right)$. Similarly, the received signal of user 2 can be written as

$$
\boldsymbol{y}_{2}=\boldsymbol{h}_{2}\left(\sqrt{1-\alpha} s_{1}+\sqrt{\alpha} s_{2}\right)+\boldsymbol{n}_{2},
$$

Where $\boldsymbol{n}_{2}$ denotes an AWGN vector and $\boldsymbol{n}_{2} \sim C N\left(0, \sigma_{2}^{2} \boldsymbol{I}\right)$.

As aforementioned, since NOMA scheme permits signals belong to different users transmit on shared resource, this introduces strong inter-user interference. Thus, two key points must be taken into account in P-NOMA, one is the power allocation which can effectively control the introduced inter-user interference. In this work, we employ weighted sum-rate maximization as the power distribution criterion, and the correspondin power allocation algorithm can be found in the Appendix. The other is the efficient multiuser detection (MUD) algorithm which will be discussed in the following section.

\subsection{Detection algorithm for P-NOMA system}

In this section, we give a description of the SIC based detection for P-NOMA. The far user performs the linear minimum mean square error (LMMSE) filtering and considers the signal of near user as noise, while the near user needs to cancel the signal of far user to achieve its capacity.

a) Detection of the far user

For the far user, i.e. user 1, a LMMSE filtering algorithm can be employed which considers $s_{2}$ as noise. Thus, the estimate of the combined signal $s$, denoted as $\hat{s}$, can be written as

$$
\hat{s}=\mathbf{W}_{m m s e} \boldsymbol{y}_{1}=\mathbf{W}_{m m s e}\left(\boldsymbol{h}_{1} \sqrt{1-\alpha} s_{1}+\left(\boldsymbol{h}_{1} \sqrt{\alpha} s_{2}+\boldsymbol{n}_{1}\right)\right),
$$

where $\mathbf{W}_{\text {mmse }}=\left(\boldsymbol{h}_{1}^{H} \boldsymbol{h}_{1}+N_{1} \mathbf{I}\right)^{-1} \boldsymbol{h}_{1}^{H}$ and $\mathbf{I}$ is the identity matrix. The estimate of $s_{1}$, denoted as $\hat{s}_{1}$, can be written as

$$
\hat{s}_{1}=\frac{\hat{s}}{\sqrt{1-\alpha}}
$$

b) Detection of the near user

For the near user (user 2), a SIC algorithm is employed. First, $s_{1}$ can be detected while considers $s_{2}$ as noise, as shown in Fig. 2. After soft demodulation and decoding, the reconstructed soft symbol $\overline{s_{1}}$ associated to $s_{1}$ is cancelled from the combined signal $\hat{s}$. At last, we perform the soft detection and decoding for $s_{2}$. Let $\hat{s}_{2}$ denote the estimate of $s_{2}$ while $s_{2}^{\prime}$ 
denote the hard decision of $s_{2}$.

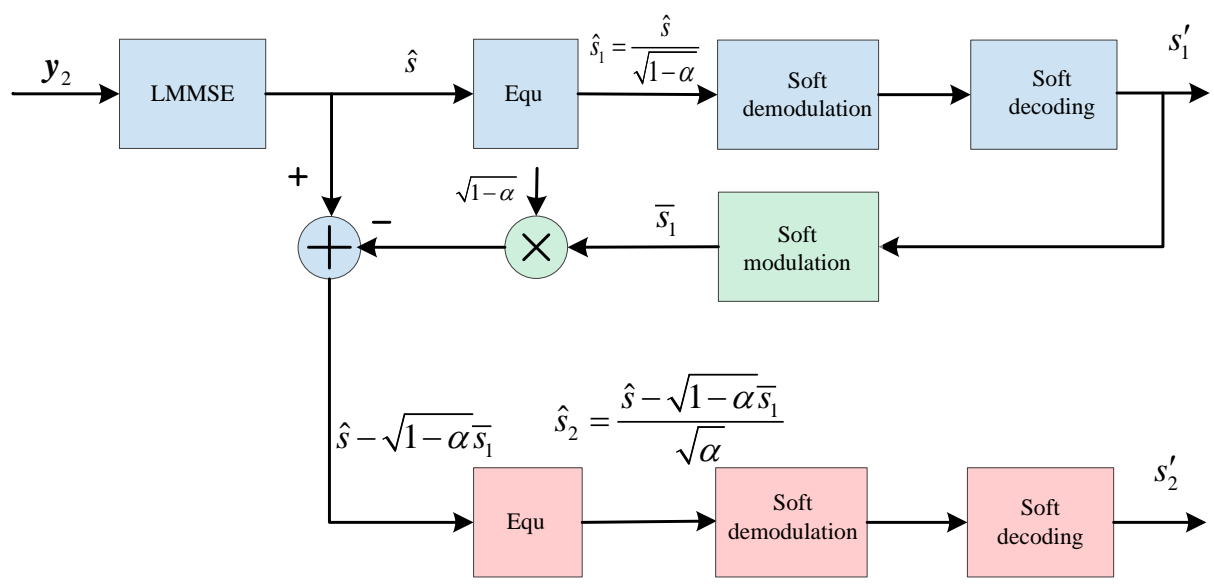

Fig. 2. The detection of near user (user 2)

The detection of $s_{2}$ can be summarized as algorithm 1 .

Algorithm1: Detection of the near user with SIC

1: Step 1: Calculate the estimator $\hat{s}$ of the combined signal $s$ with LMMSE filtering,

$$
\hat{s}=\mathbf{W}_{m m s e} \boldsymbol{y}_{2}=\sqrt{1-\alpha} \hat{s}_{1}+\sqrt{\alpha} \hat{s}_{2}
$$

where $\mathbf{W}_{\text {mmse }}=\left(\boldsymbol{h}_{2}^{H} \boldsymbol{h}_{2}+\sigma_{2}^{2} \mathbf{I}\right)^{-1} \boldsymbol{h}_{2}^{H}$.

2: Step 2: Calculate the estimator $\hat{s}_{1}$ as follows

$$
\hat{s}_{1}=\frac{\hat{s}}{\sqrt{1-\alpha}} \text {. }
$$

3: Step 3: Soft demodulation, soft decoding and reconstruct $s_{1}$. Assuming that the bit-LLR after demodulation and decoding can be denoted as $L_{b}, b=0,1, B-1$. Then the reconstructed soft symbol

$$
\overline{S_{1}}=\sum_{\theta \in \mathcal{A}} \theta \prod_{b=0}^{B-1}\left(1+\left(2 c_{b}-1\right) \tanh \left(\frac{L_{b}}{2}\right)\right) .
$$

Where $B$ bits tube $\left[c_{0}, c_{1}, \cdots, c_{B-1}\right]$ corresponding to constellation point $\theta$ which belongs to constellation $\mathcal{A}$.

4: Step 4: SIC procedure. The estimate of $s_{2}$ can be expressed as

$$
\hat{s}_{2}=\frac{\hat{s}-\sqrt{1-\alpha} \bar{s}_{1}}{\sqrt{\alpha}} .
$$

This can also be simplified as $\hat{s}_{2}=\left(\frac{\hat{s}}{\sqrt{1-\alpha}}-\bar{s}_{1}\right) \frac{\sqrt{1-\alpha}}{\sqrt{\alpha}}=\sqrt{\frac{1-\alpha}{\alpha}}\left(\hat{s}_{1}-\bar{s}_{1}\right)$. After soft demodulation and decoding, we can obtain the hard-decision output $s_{2}^{\prime}$. 


\section{Proposed MLMR coded P-NOMA for multiple antenna system}

In LTE system, multiple antenna technique is widely employed to achieve the multiplexing gain and diversity gain. Thus, it is interesting to combine MIMO with NOMA technique for multiuser system. As we can see, there exist two types interference in the MIMO-NOMA system, one is inter-user interference, the other is inter-stream interference within MIMO system. The former can be well overcome by P-NOMA with SIC detection at the receiver side as show section 2. In this section, we present a scheme that combines QO-STBC and SIC-based detector to eliminate inter-stream interference for MIMO system. On the whole, we proposed a joint space-time and power domain transmission scheme for MIMO-NOMA system.

\subsection{Structure of the proposed QO-STBC coded P-NOMA}

When the BS equipped with 2 antennas, Alamouti's coding scheme can achieve full diversity gain [17]. We take the BS equipped with 3 antennas for example. Fig. 3 shows the transmit matrix of existing scheme that the BS transmits multiple data-streams in Vertical Bell Labs Layered Space Time (VBLAST) manner, while Fig. $\mathbf{4}$ is the proposed scheme that the BS transmits multiple data streams in QO-STBC manner. In both figures, the subscript $i$ denotes the $i$-th user while the subscript $j$ denotes the $j$-th data-stream belongs to user $i$. After reasonable power allocation, these two space-time coded data blocks superimposed over the same resources and transmitted. As we can see in Fig. 3, in the traditional VBLAST coded P-NOMA case, each user can transmit 9 symbols over 3 antennas and 3 time slots. Thus, user1 and user 2 altogether can support 18 symbols over the resource above. In Fig. 4, each user in the proposed QO-STBC coded P-NOMA scheme can transmit 5 symbols over the same resource as aforementioned. For comparison purpose, we need to adjust the channel coding rates to make these two schemes have the same spectral efficiency. Moreover, the above two schemes are of the following common characteristics: 1) both schemes are power-domain NOMA, i.e., users are separated by different power; 2) both schemes can use SIC-based detector.

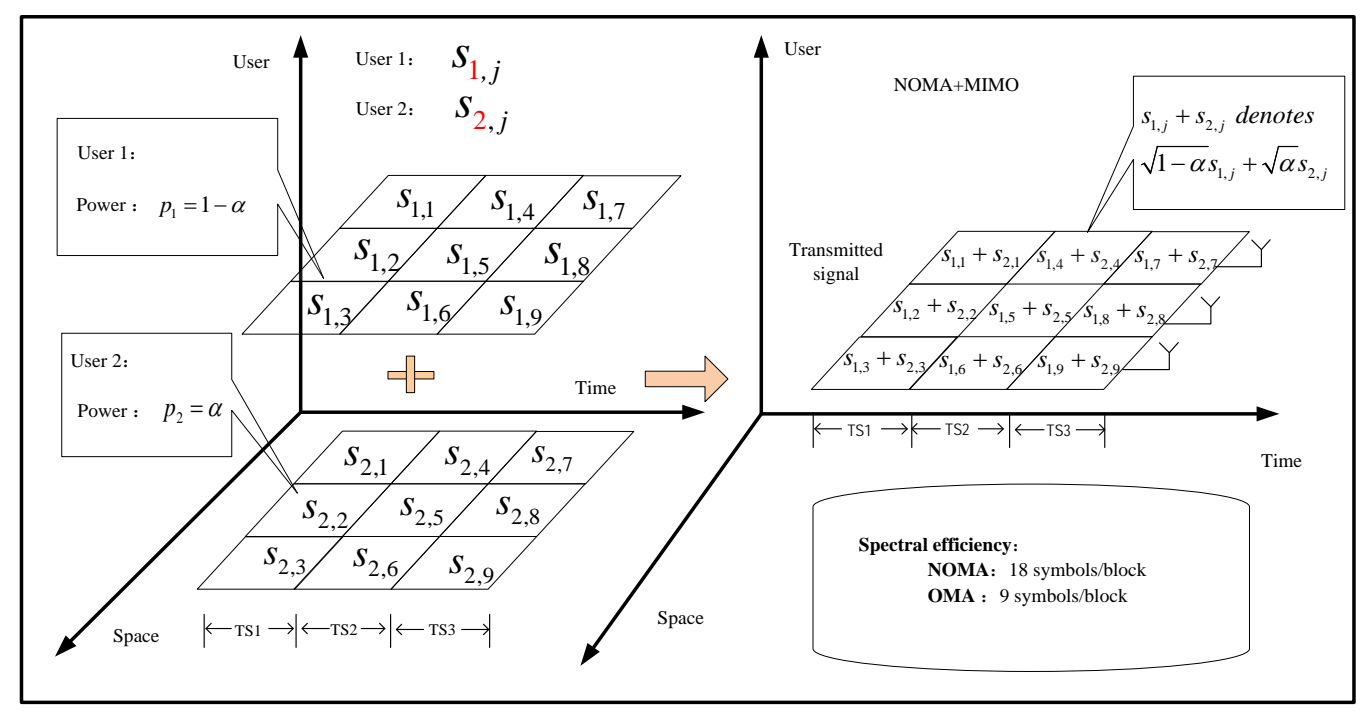

Fig. 3. Traditional VBLAST coded P-NOMA 


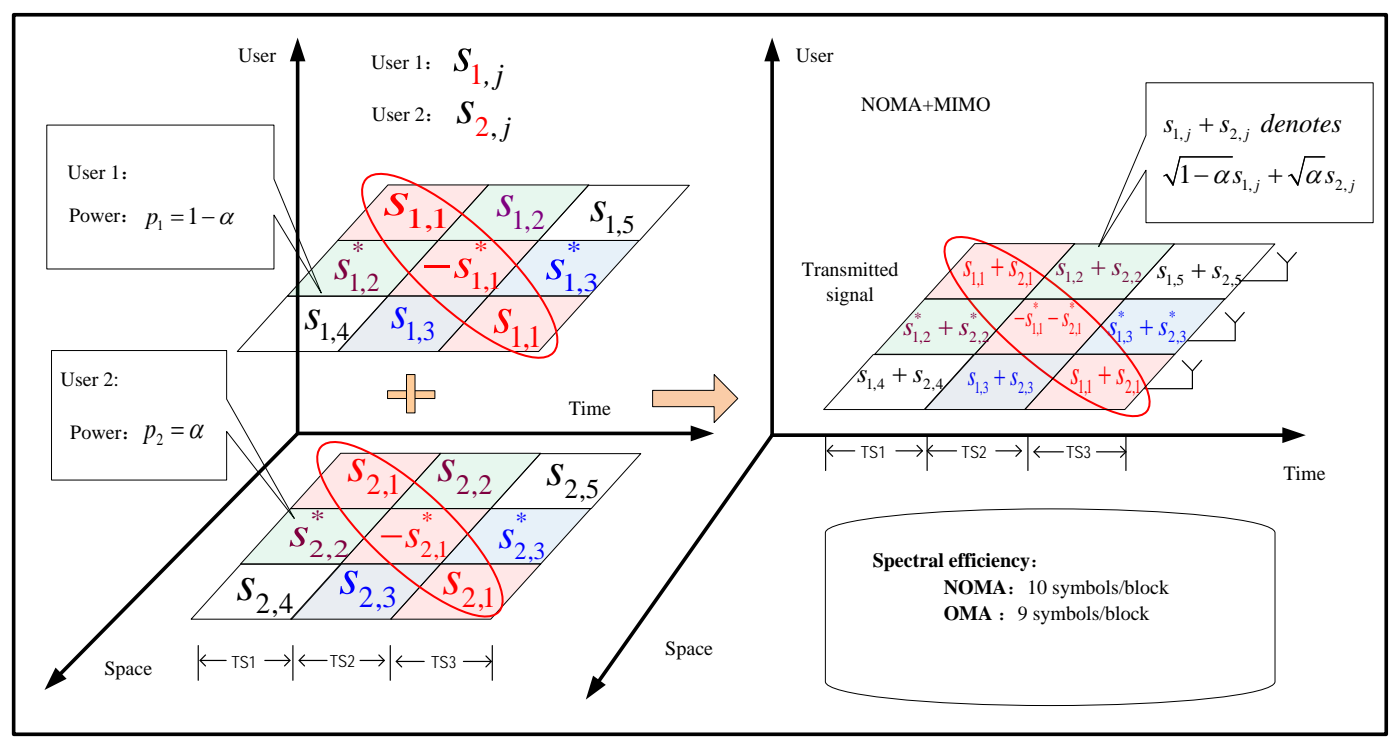

Fig. 4. Proposed QO-STBC coded P-NOMA

The difference between these two P-NOMA schemes is the former scheme transmits intra-user data-streams in VBLAST manner while the later scheme operating in QO-STBC manner.As we can see, both schemes existing inter-user interference and intra-user interference, as a result we need to use the SIC-based detector twice, the first for the data separation of NOMA while the second for the data-stream separation of MIMO. For both schemes, the far-user suffers the same interference while the near-user benefit from interference cancellation.

\subsection{The designation of QO-STBC}

As mentioned in section 3.1, we proposed QO-STBC code instead of VBLAST code in order to improve the BER performance of MIMO-NOMA system. For MIMO spatial multiplexing system, the diversity order $N_{D}$ of the $i$-th data-layer after SIC-based detection can be written as [18]

$$
N_{D}=N_{r}-N_{t}+i
$$

where $N_{r}$ denotes the number of receive antennas, $N_{t}$ denotes the number of transmit antennas. According to formula (6), for VBLAST system, the diversity order of the first layer is the lowest among all data-layers which leads to error propagation (EP) and thus degraded BER performance. In another word, due to the EP, the performance of the following data layers depend on that of the first layer to a large extend. To overcome this problem, we resort to the design of unequal transmit diversity order which can improve the performance of the first-layer which thus can improve/balance the performance of all data layers. The main ideal is to adjust the transmit diversity order to make the diversity order of all data layers have about the same post-detection diversity order. In this subsection, two QO-STBC schemes are proposed for $3 \times 3$ and $4 \times 4$ MIMO system as shown in Fig. 5(a) and Fig. 5(b) respectively [19-20]. Let $N_{t}$ denote the number of transmit antenna and $\mathbf{G}_{N_{t}}$ denote the corresponding 
QO-STBC transmit matrix. Assuming $\mathbf{G}_{N_{t}}$ transmit $M$ modulated symbols in $T$ time-slot, thus the coding rate of $\mathbf{G}_{N_{t}}$ is $R_{\mathrm{G}}=M / T$. For $\mathbf{G}_{3}$ in Fig. 5(a), it transmits $M=5$ symbols during $T=3$ time slots. We classify these $M$ symbols into 3 data layers which have about the same post-detection diversity order. It is needed to point out that we define data-layer as the set of symbols are of the same transmit diversity order in this paper. Therefore, $x_{1}$ belongs to layer 1 , $x_{2}$ and $x_{3}$ belongs to layer 2 while $x_{4}$ and $x_{5}$ belong to layer 3. Under ideal SIC-based detection, take $\mathbf{G}_{3}$ for example, the post-detection diversity order of $x_{1}$ equals to $N_{p}=4$ since its transmit diversity order is 3 as shown in Table 1. Similarly, the post-detection diversity order of both $x_{2}$ and $x_{3}$ are 4 and they also equal to 4 for $x_{4}$ and $x_{5}$. All these three data layers have the same post-detection diversity order which means these data layers exhibit about the same error performance thus leading to the improved overall system performance. Similarly, for QO-STBC $\mathbf{G}_{4}$, we have the similar conclusions, as shown in Table 2.

$$
\mathbf{G}_{3}=\left[\begin{array}{ccc}
x_{1} & x_{2}^{*} & x_{5} \\
x_{2} & -x_{1}^{*} & x_{3} \\
\hdashline x_{4} & x_{3}^{*} & x_{1}
\end{array}\right]
$$

(a) Transmit matrix for $N_{t}=3$

$$
\mathbf{G}_{4}=\left[\begin{array}{cc:cc}
x_{1} & x_{2}^{*} & x_{5} & x_{8}^{*} \\
x_{2} & -x_{1}^{*} & x_{3} & x_{6}^{*} \\
\hdashline x_{5} & x_{3}^{*} & x_{1} & x_{4}^{*} \\
x_{7} & x_{6}^{*} & x_{4} & -x_{1}^{*}
\end{array}\right]
$$

(b) Transmit matrix for $N_{t}=4$

Fig. 5. Tranmit pattern matrix of QO-STBC with 3 or 4 antennas

Table 1. Post-detection diversity order $N_{p}$ under SIC for $\mathbf{G}_{3}$

\begin{tabular}{|c|c|c|c|c|}
\multicolumn{7}{c}{ Table 1. Post-detection diversity order $N_{p}$ under SIC for $\mathbf{G}_{3}$} \\
\hline Data-layer & Data & $\begin{array}{c}\text { Transmit } \\
\text { diversity order }\end{array}$ & $N_{D}$ & $N_{P}$ \\
\hline \hline$i=1$ & $x_{1}$ & 3 & 1 & 4 \\
\hline$i=2$ & $x_{2}, x_{3}$ & 2 & 2 & 4 \\
\hline$i=3$ & $x_{4}, x_{5}$ & 1 & 3 & 4 \\
\hline
\end{tabular}

Table 2. Post-detection diversity order $N_{p}$ under SIC for $\mathbf{G}_{4}$

\begin{tabular}{|c|c|c|c|c|}
\hline Data-layer & Data & $\begin{array}{c}\text { Transmit } \\
\text { diversity order }\end{array}$ & $N_{\mathrm{D}}$ & $N_{\mathrm{p}}$ \\
\hline \hline$i=1$ & $x_{1}$ & 4 & 1 & 5 \\
\hline$i=2$ & $x_{2}, x_{3}, x_{4}, x_{5}, x_{6}$ & 2 & 2 & 4 \\
\hline$i=3$ & $x_{7}, x_{8}$ & 1 & 3 & 4 \\
\hline
\end{tabular}




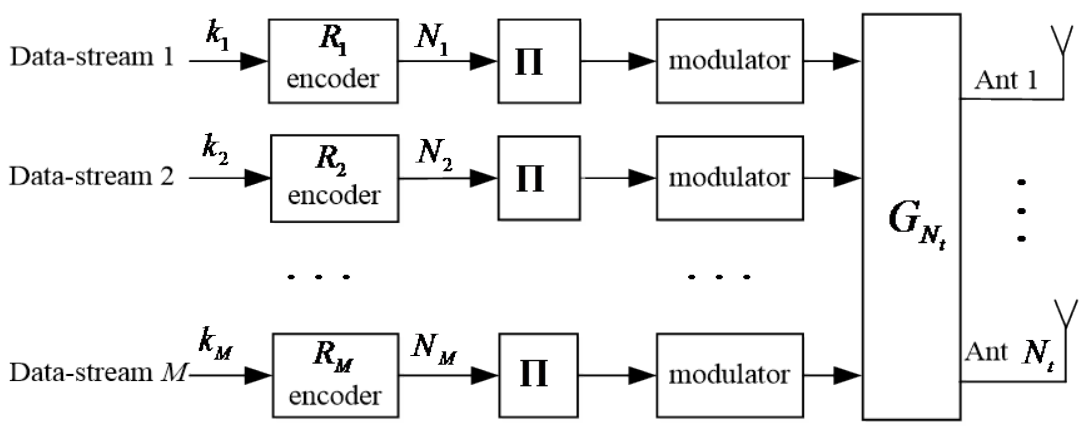

(a) Transceiver block diagram of MLMR coded QO-STBC system

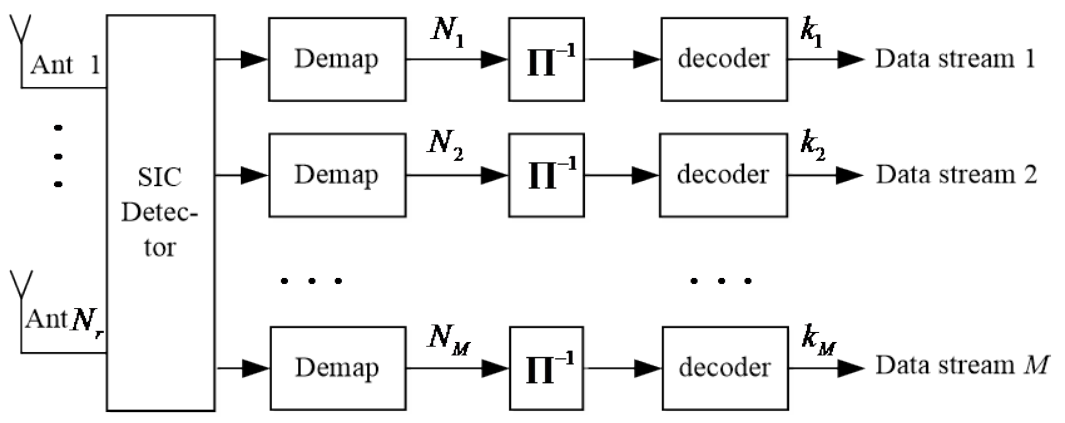

(b) Receiver block diagram of MLMR coded QO-STBC system

Fig. 6. Transmitter and receiver block diagram of MLMR coded QO-STBC

\subsection{System model of the proposed MLMR coded P-NOMA}

The block diagram of the transmitter of the proposed MLMR coded QO-STBC scheme is shown as in Fig. 6(a). Assuming that $M$ data streams $\mathbf{b}_{1}, \mathbf{b}_{2}, \cdots, \mathbf{b}_{M}$, each of which contains $K_{i}$ bits, is encoded with a turbo code with coding rate $R_{i}, \mathbf{0}<R_{i}<\mathbf{1}$, respectively, where $\mathbf{b}_{i}=\left[b_{i, 1}, b_{i, 2}, \cdots, b_{i, K_{i}}\right]$ for $\mathbf{1} \leq \boldsymbol{i} \leq \boldsymbol{M}$. The output of the turbo encoder can be expressed as $\mathbf{c}_{i}=\left[c_{i, 1}, c_{i, 2}, \cdots, c_{i, N_{i}}\right]$ for $\mathbf{1} \leq \boldsymbol{i} \leq \boldsymbol{M}$, where the length of the codeword is $N_{i}$. The turbo coding rate of the $i$-th data-stream is $R_{i}=K_{i} / N_{i}$. After scrambling and modulation, $M$ modulated symbols each of which picked up from $M$ data streams are divided into $L$ data layers and then are mapped onto the QO-STBC matrix $\mathbf{G}_{T_{x}}$, as shown in Fig. 5, at last transmit over $T_{x}$ antennas within $T$ time slots. For simplicity, all data layers employ the same modulation constellation, denoted as $\mathcal{A}$, the size of which is $|\mathcal{A}|$. Moreover, suppose that $\boldsymbol{m}_{i}$ data streams belong to the $i$-th data-layer and $\sum_{i=1}^{L} m_{i}=M$, it is naturally these $m_{i}$ data streams can share a common turbo encoder/decoder. The overall coding rate of the MLMR can be expressed as

$$
R_{c}=\frac{1}{M} \sum_{i=1}^{L} m_{i} R_{i}
$$

Suppose that each UE equipped with $N_{r}$ antennas, since both users share the same form of baseband receive signal, we omit the use-index for simplicity. The baseband signal at user $i$, 
$i=1,2$, can be described by

$$
\mathbf{Y}=\sqrt{\frac{\rho}{N_{t}}} \mathbf{H S}+\mathbf{V}
$$

$\mathbf{Y}=\left[\boldsymbol{y}_{1}, \boldsymbol{y}_{2}, \cdots, \boldsymbol{y}_{t}, \cdots, \boldsymbol{y}_{T}\right]$ is the received signal matrix, $\mathbf{S}=\left[\boldsymbol{s}_{1}, \boldsymbol{s}_{2}, \cdots, \boldsymbol{s}_{t}, \cdots, \boldsymbol{s}_{T}\right]$ is the QO-STBC matrix, $\mathbf{H}$ is the channel coefficients matrix from the BS to the $i$-th user with $h_{i, j} \sim C N(0,1)$. $\mathbf{V}$ is the noise matrix at the destination with entity $V_{i, j} \sim C N(0,1) . T$ is the QO-STBC signaling period. In formula (8), transmit matrix $\mathbf{S}=\mathbf{G}_{N_{t}}=\psi(\boldsymbol{X}(j))$, where $\boldsymbol{X}(j)=\left[x_{1, j}, x_{2, j}, \cdots, x_{M, j}\right]^{T}$ denote the transmitted $M$ symbols during $j$-th code block, $1 \leq j \leq\left[N / \log _{2}|\mathcal{A}|\right] .|\mathcal{A}|$ denotes the size of the constellaiton. $\psi(\cdot)$ denotes the QO-STBC mapping function from $M$ symbols to code matrix $\mathbf{G}_{N_{t}}$. Let $\rho$ denote the average single user signal-to-noise ratio (SNR) per receive antenna for fair comparison purpose.

\subsection{Two stage SIC-based detection for the proposed scheme}

As aforementioned, QO-STBC coded P-NOMA system need to perform SIC twice, the first for data separation of P-NOMA, the second for MIMO. The detection of P-NOMA has been described in section 2.2. In this section, we dedicate to the SIC/OSIC-based detection of QO-STBC. For $N_{t}=N_{r}=3$, i.e. $\mathbf{G}_{3}$ is employed as transmit matrix, the received signal can be expressed as

$$
\left[\begin{array}{lll}
y_{11} & y_{12} & y_{13} \\
y_{21} & y_{22} & y_{23} \\
y_{31} & y_{32} & y_{33}
\end{array}\right]=\sqrt{\frac{\rho}{3}}\left[\begin{array}{lll}
h_{11} & h_{12} & h_{13} \\
h_{21} & h_{22} & h_{23} \\
h_{31} & h_{32} & h_{33}
\end{array}\right]\left[\begin{array}{ccc}
x_{1} & x_{2}^{*} & x_{5} \\
x_{2} & -x_{1}^{*} & x_{3} \\
x_{4} & x_{3}^{*} & x_{1}
\end{array}\right]+\left[\begin{array}{ccc}
v_{1,1} & v_{1,2} & v_{1,3} \\
v_{2,1} & v_{2,2} & v_{2,3} \\
v_{3,1} & v_{3,2} & v_{3,3}
\end{array}\right] .
$$

The above equation can be converted to the following form equivalently.

$$
\left[\begin{array}{l}
y_{1,1} \\
y_{2,1} \\
y_{3,1} \\
y_{1,2}^{*} \\
y_{2,2}^{*} \\
y_{3,2}^{*} \\
y_{1,3}^{*} \\
y_{2,3} \\
y_{3,3}
\end{array}\right]=\sqrt{\frac{\rho}{3}}\left[\begin{array}{ccccc}
h_{1,1} & h_{1,2} & 0 & h_{1,3} & 0 \\
h_{2,1} & h_{2,2} & 0 & h_{2,3} & 0 \\
h_{3,1} & h_{3,2} & 0 & h_{3,3} & 0 \\
-h_{1,2}^{*} & h_{1,1}^{*} & h_{1,3}^{*} & 0 & 0 \\
-h_{2,2}^{*} & h_{2,1}^{*} & h_{2,3}^{*} & 0 & 0 \\
-h_{3,2}^{*} & h_{3,1}^{*} & h_{3,3}^{*} & 0 & 0 \\
h_{1,3} & 0 & h_{1,2} & 0 & h_{1,1} \\
h_{2,3} & 0 & h_{2,2} & 0 & h_{2,1} \\
h_{3,3} & 0 & h_{3,2} & 0 & h_{3,1}
\end{array}\right]\left[\begin{array}{c}
x_{1} \\
x_{2} \\
x_{3} \\
x_{4} \\
x_{5}
\end{array}\right]+\left[\begin{array}{c}
v_{1,1} \\
v_{1,2} \\
v_{1,3} \\
v_{2,1}^{*} \\
v_{2,2}^{*} \\
v_{2,3}^{*} \\
v_{3,1} \\
v_{3,2} \\
v_{3,3}
\end{array}\right] .
$$

Similarly, when $N_{t}=N_{r}=4$, i.e. $\mathbf{G}_{4}$ is employed, the received signal at receiver can also be written as 


$$
\left[\begin{array}{l}
y_{11} \\
y_{21} \\
y_{31} \\
y_{41} \\
y_{12}^{*} \\
y_{22}^{*} \\
y_{32}^{*} \\
y_{42}^{*} \\
y_{13} \\
y_{23} \\
y_{33} \\
y_{43} \\
y_{14}^{*} \\
y_{24}^{*} \\
y_{34}^{*} \\
y_{44}^{*}
\end{array}\right]=\sqrt{\frac{\rho}{4}}\left[\begin{array}{cccccccc}
h_{11} & h_{12} & 0 & 0 & h_{13} & 0 & h_{14} & 0 \\
h_{21} & h_{22} & 0 & 0 & h_{23} & 0 & h_{24} & 0 \\
h_{31} & h_{32} & 0 & 0 & h_{33} & 0 & h_{34} & 0 \\
h_{41} & h_{42} & 0 & 0 & h_{43} & 0 & h_{44} & 0 \\
-h_{12}^{*} & h_{11}^{*} & h_{13}^{*} & 0 & 0 & h_{14}^{*} & 0 & 0 \\
-h_{22}^{*} & h_{21}^{*} & h_{23}^{*} & 0 & 0 & h_{24}^{*} & 0 & 0 \\
-h_{32}^{*} & h_{31}^{*} & h_{33}^{*} & 0 & 0 & h_{34}^{*} & 0 & 0 \\
-h_{42}^{*} & h_{41}^{*} & h_{43}^{*} & 0 & 0 & h_{44}^{*} & 0 & 0 \\
h_{13} & 0 & h_{12} & h_{14} & h_{11} & 0 & 0 & 0 \\
h_{23} & 0 & h_{22} & h_{24} & h_{21} & 0 & 0 & 0 \\
h_{33} & 0 & h_{32} & h_{34} & h_{31} & 0 & 0 & 0 \\
h_{43} & 0 & h_{42} & h_{44} & h_{41} & 0 & 0 & 0 \\
-h_{14}^{*} & 0 & 0 & h_{13}^{*} & 0 & h_{12}^{*} & 0 & h_{11}^{*} \\
-h_{24}^{*} & 0 & 0 & h_{23}^{*} & 0 & h_{22}^{*} & 0 & h_{21}^{*} \\
-h_{34}^{*} & 0 & 0 & h_{33}^{*} & 0 & h_{32}^{*} & 0 & h_{31}^{*} \\
-h_{44}^{*} & 0 & 0 & h_{43}^{*} & 0 & h_{42}^{*} & 0 & h_{41}^{*}
\end{array}\right]\left[\begin{array}{c}
x_{1} \\
x_{2} \\
x_{3} \\
x_{4} \\
x_{5} \\
x_{6} \\
x_{7} \\
x_{8}
\end{array}\right]+\left[\begin{array}{c}
v_{11} \\
v_{21} \\
v_{31} \\
v_{41} \\
v_{12}^{*} \\
v_{22}^{*} \\
v_{32}^{*} \\
v_{42}^{*} \\
v_{13} \\
v_{23} \\
v_{33} \\
v_{43} \\
v_{14}^{*} \\
v_{24}^{*} \\
v_{34}^{*} \\
v_{44}^{*}
\end{array}\right] .
$$

In this manner, for arbitrary QO-STBC system with $N_{r}>R_{G}$, we can obtain the equivalent channel matrix as shown above. Then zero forcing (ZF) or SIC-based detection algorithm can be adopted. For example, ordered SIC-based LMMSE detection algorithm [16, 21] can be summarized as follows.

Step1: Initialization. For the detection of the first layer, let

$$
\mathbf{H}^{1}=\mathbf{H}, \boldsymbol{y}_{t}^{1}=\boldsymbol{y}_{t} .
$$

Step2: For the detection of the $i$-th layer, if LMMSE is employed, then the LMMSE weighting matrix can be expressed as

$$
\mathbf{W}^{i}=\left(\left(\mathbf{H}^{i}\right)^{H}\left(\mathbf{H}^{i}\right)+\sigma^{2} \mathbf{I}\right)^{-1}\left(\mathbf{H}^{i}\right)^{H} .
$$

We need to order the data-layer according to the SNR, which is equivalent to the norm of each column vector of $\mathbf{W}^{i}$. For example, we choose $k_{i}$ as the current layer if

$$
\begin{gathered}
k_{i}=\underset{j \notin\left\{1,2, \cdots, N_{t}-i+1\right\}}{\arg \min }\left\|\left(\mathbf{W}^{i}\right)_{j}\right\|^{2}, \\
\boldsymbol{w}_{k_{i}}=\left(\mathbf{W}^{i}\right)_{j} .
\end{gathered}
$$

The output soft symbol can be expressed as

$$
\hat{x}_{k_{i}}=\mathcal{Q}\left(\boldsymbol{w}_{k_{i}}^{H} \boldsymbol{y}_{t}^{i}\right) .
$$

where $\mathcal{Q}(\cdot)$ denotes the slice operation.

Step3: Interference cancellation. Subtract the detected symbol from $y_{t}^{i}$, we have the residual signal as 


$$
\boldsymbol{y}_{t}^{i+1}=\boldsymbol{y}_{t}^{i}-\boldsymbol{h}_{k_{i}} \hat{x}_{k_{i}} .
$$

Where $\boldsymbol{h}_{k_{i}}$ denotes the $k_{i}$-th column of channel matrix $\mathbf{H}^{i}$. We denote the residual channel matrix after eliminating the $k_{i}$-th column as

$$
\mathbf{H}^{i+1}=\mathbf{H}_{\bar{k}_{i}}^{i} .
$$

Step 4: Set $i=i+1$, repeat from step 2 to step 3 until $i=N_{t}$.

\subsection{Optimization of the coding rate of MLMR}

QO-STBC with well-designed transmit diversity order is convergence-aware and can achieve better performance over the VBLAST scheme. However, it can also be further improved by coding multiple layers with multiple rate (MLMR) channel codes, the diagram of which is shown as in Fig. 5(a). The proposed MLMR coded P-NOMA scheme exhibits better performance especially in the $\mathbf{G}_{4}$ system in which the post-detection diversity order is no longer keep equal strictly as shown in Table 2 . In this section, we propose to optimize the channel coding rate of MLMR according to the post-detection SNR which can efficiently eliminate the residual interference and thus can further balance the error performance of different data layers. To calculate the post-detection SNR of the LMMSE detection, we consider the correlation matrix $\mathbf{R}_{e}$ of the estimation error vector $\boldsymbol{e}=\boldsymbol{x}-\hat{\boldsymbol{x}} \cdot \mathbf{R}_{e}$ can be written as bellows

$$
\begin{aligned}
\mathbf{R}_{e} & =E\left[|\boldsymbol{x}-\hat{\boldsymbol{x}}|^{2}\right]=E\left[\left(\boldsymbol{x}-\mathbf{W}^{H} \boldsymbol{y}\right)\left(\boldsymbol{x}-\mathbf{W}^{H} \boldsymbol{y}\right)^{H}\right] \\
& =\mathbf{R}_{x x}-\mathbf{R}_{\boldsymbol{y x}}^{H} \mathbf{R}_{y \boldsymbol{y}}^{-1} \mathbf{R}_{\boldsymbol{y x}},
\end{aligned}
$$

where $\mathbf{R}_{y x}-\mathbf{R}_{y y} \mathbf{W}=\mathbf{0}$, and

$$
\left\{\begin{array}{l}
\mathbf{R}_{x x}=\mathbf{I} \\
\mathbf{R}_{y x}=E\left[(\mathbf{H} \boldsymbol{x}+\boldsymbol{n}) \boldsymbol{x}^{H}\right]=\mathbf{H R}_{x x}=\mathbf{H} \\
\mathbf{R}_{y y}=E\left[(\mathbf{H} \boldsymbol{x}+\boldsymbol{n})(\mathbf{H} \boldsymbol{x}+\boldsymbol{n})^{H}\right]=\mathbf{H} \mathbf{R}_{x x} \mathbf{H}^{H}+\sigma^{2} \mathbf{I}_{N_{t}}=\mathbf{H} \mathbf{H}^{H}+\sigma^{2} \mathbf{I}_{N_{t}}
\end{array} .\right.
$$

Combining (16) into (15), $\mathbf{R}_{e}$ can be written as

$$
\begin{aligned}
\mathbf{R}_{e} & =\mathbf{I}_{N_{t}}-\mathbf{H}^{H}\left(\mathbf{H H}^{H}+\sigma^{2} \mathbf{I}_{N_{t}}\right)^{-1} \mathbf{H} \\
& =\mathbf{I}_{N_{t}}-\left(\mathbf{H}^{H} \mathbf{H}+\sigma^{2} \mathbf{I}_{N_{t}}\right)^{-1} \mathbf{H}^{H} \mathbf{H} .
\end{aligned}
$$

According to orthogonal principle, the power of the estimator $\hat{x}_{i}$ is $E\left(\left|\hat{x}_{i}\right|^{2}\right)=E\left(\left|x_{i}\right|^{2}\right)-E\left(\left|e_{i}\right|^{2}\right)$, thus the post-detection SNR of $x_{i}$ can be written as

$$
\begin{aligned}
p_{i} & =\frac{E\left(\left|\hat{x}_{i}\right|^{2}\right)}{E\left(\left|e_{i}\right|^{2}\right)}=\frac{E\left(\left|x_{i}\right|^{2}\right)-E\left(\left|e_{i}\right|^{2}\right)}{E\left(\left|e_{i}\right|^{2}\right)} \\
& =\frac{1}{\left(\mathbf{R}_{e}\right)_{i i}}-1,
\end{aligned}
$$

where $\left(\mathbf{R}_{e}\right)_{i i}$ denotes the $i$-th diagonal entity of matrix $\mathbf{R}_{e}$. According to (15), we can obtain the post-detection SNR $p_{i}$ for $1 \leq i \leq N_{t}$ 。

The coding rate optimization algorithm for MLMR can be summarized as Algorithm 2. 
Algorithm 2: Coding rate optimization of MLMR

Step 1: Initialization. Let $i=1$, and $\mathbf{H}^{1}=\mathbf{H}$.

Step 2: Compute the post-detection SNR $p_{1}$ for the first data-layer according to (14) and (15).

Step 3: Dimension reduction. Let $\boldsymbol{h}_{k_{i}}$ denotes the $i$-th column of the channel matrix $\mathbf{H}^{i}$.

After eliminating the $k_{i}$-th column, $\mathbf{H}^{i}$ reduced to

$$
\mathbf{H}^{i+1}=\mathbf{H}_{\bar{k}_{i}}^{i}
$$

Step 4: Repeat step 2 step 3. and set $i=i+1$ until all $p_{i}^{\text {'s }, ~} 1 \leq i \leq N_{t}$, are computed.

Step 5: Under the sum-rate constrain, choose component coding rate for each data layer.

Next, we take $\mathbf{G}_{3}$ for example to show how to perform step 5 in detail. According to step 1 step 4, we can obtain the achievable rate corresponding to the post-detection SNR of all three data layers and the sum rate as shown in Fig. 7. Take VBLAST scheme with turbo coding rate $R_{c}=0.75$ and quadrature phase shift keying (QPSK) as baseline, the spectral efficiency of which is $3 \times 2 \times 0.75=4.5 b i t s / \mathrm{s} / \mathrm{Hz}$. For QO-STBC coded scheme, we choose the component coding rate corresponding to which has the sum rate $4.5 \mathrm{bits} / \mathrm{s} / \mathrm{Hz}$. Eventually, we have $C_{1}=3.36 \mathrm{bits} / \mathrm{s} / \mathrm{Hz}, \mathrm{C}_{2}=2.95 \mathrm{bits} / \mathrm{s} / \mathrm{Hz}$ and $\mathrm{C}_{3}=2.12 \mathrm{bits} / \mathrm{s} / \mathrm{Hz}$. The corresponding sum rate is $C=\left(C_{1}+2 C_{2}+2 C_{3}\right) / 3=4.5 \mathrm{bits} / \mathrm{s} / \mathrm{Hz}$. Therefore, we choose $R_{1}=C_{1} / 4=0.84$, data layer 2 with coding rate $R_{2}=C_{2} / 4=0.7375$ and data layer 3 with coding rate $R_{3}=C_{3} / 4=0.53$, all data layers with 16-ary quadrature amplitude modulation (16QAM). The overall spectral efficiency is also $4.5 \mathrm{bit} / \mathrm{s} / \mathrm{Hz}$. Similarly, the the coding rate of $\mathbf{G}_{4}$ can be determined as the method above as shown in Fig. 8.

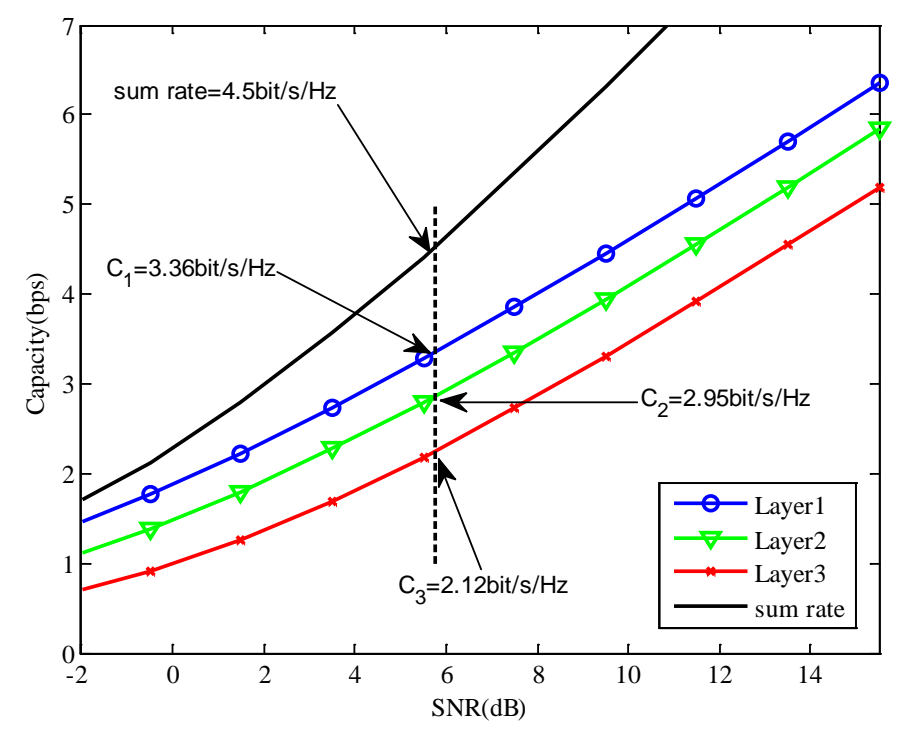

Fig. 7. The achievable channel capacity after LMMSE-OSIC detection of each data-layer of $\mathbf{G}_{3}$ and sum capacity 


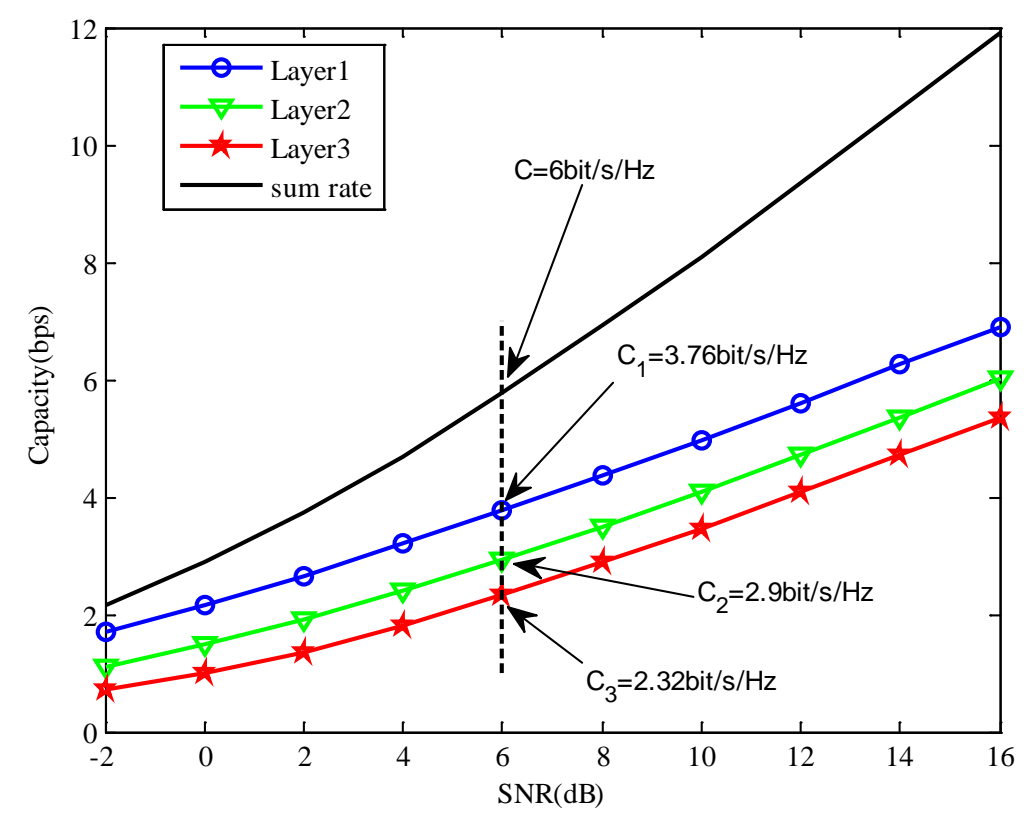

Fig. 8. The achievable channel capacity after LMMSE-OSIC detection of each data-layer of $\mathbf{G}_{4}$ and sum capacity

\section{Simulation results and analysis}

In this subsection, we simulate MLMR coded QO-STBC system with code rate optimized with the proposed algorithm 2 and make a comparison with the single rate coded QO-STBC/VBLAST system, which are denoted as MLSR-QO-STBC/MLSR-VBLAST respectively. In this paper, we assume that the channel matrix is perfectly known at the receiver side but the transmitter has only partial channel knowledge, i.e. the SNR parameter.

\subsection{BER performance comparison}

a) $\mathbf{G}_{3}$ based system

In Fig. 9, the BER performance of $\mathbf{G}_{3}$-based systems are presented which employ MLMR code and MLSR code respectively. For comparison purpose, MLSR coded VBLAST system is also considered. For MLMR coded $\mathbf{G}_{3}$ system, three turbo codes according to LTE standard [22] with optimized rate are employed. According to Algorithm 2. we have $R_{1}=0.84$, $R_{2}=0.7375, R_{3}=0.53$. The average coding rate is $R_{c}=(0.84+2 \times 0.7375+2 \times 0.53) / 5=0.675$. MLSR coded QO-STBC system employs a single turbo code of rate 0.675 and QPSK modulation scheme. VBLAST system employs a rate 0.75 turbo code and QPSK modulation. All three schemes are of the same spectral efficiency $\eta=5 / 3 \times 4 \times 0.675=3 \times 2 \times 0.75=4.5$ bits/s/Hz. As shown in Fig. 9, when LMMSE-OSIC-based detector is employed, the MLSR coded QO-STBC scheme outperforms the MLSR coded VBLAST scheme 3.4dB. Furthermore, when the proposed MLMR coded QO-STBC scheme is employed, there is a further $0.5 \mathrm{~dB}$ performance gain, and thus the overall performance gain is up to $3.9 \mathrm{~dB}$. When ZF-OSIC based 
detector is used, at the BER level of $1 \times 10^{-5}$, the MLSR coded QO-STBC outperforms the MLSR coded BLAST system 6.8dB. Similarly, when MLMR code is employed instead of MLSR code, there is another $0.5 \mathrm{~dB}$ performance gain. In this situation, the overall performance gain is up to 7.3dB over the MLSR coded VBLAST system.

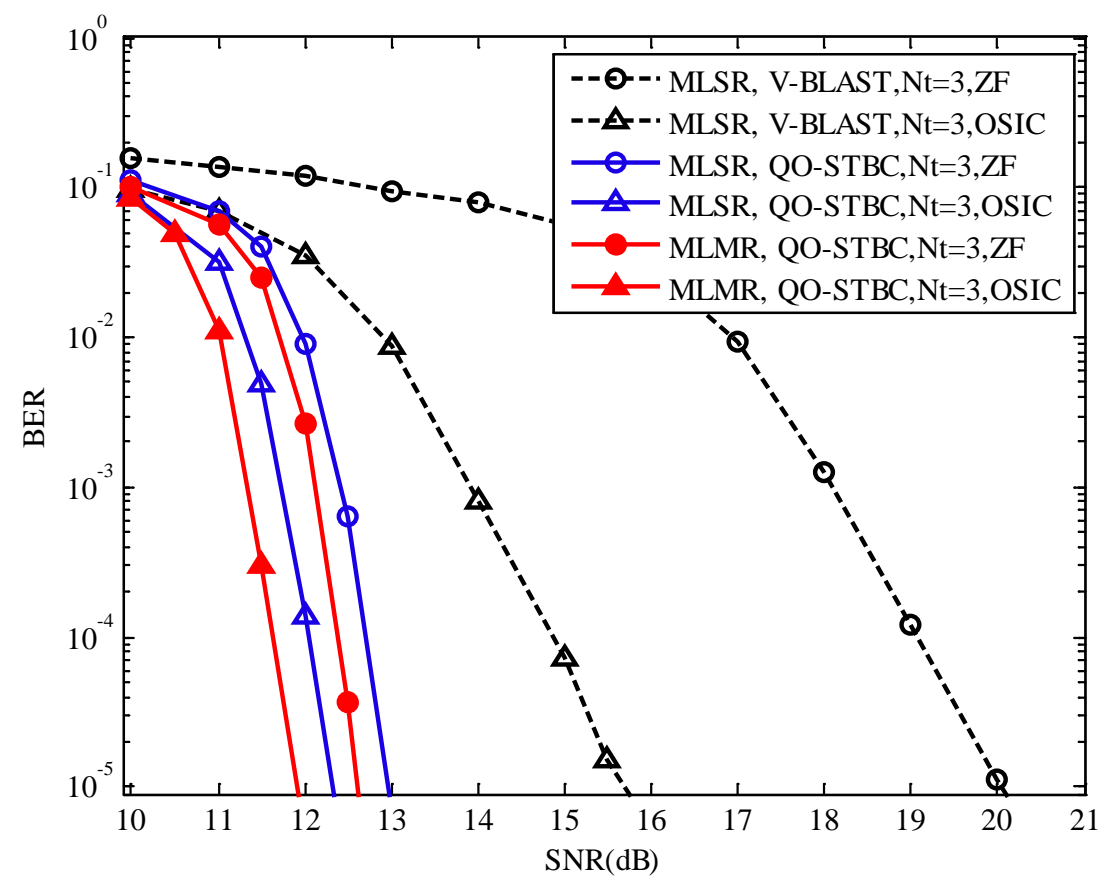

Fig. 9. Under $N_{t}=N_{r}=3$, i.e. $\mathbf{G}_{3}$, BER performance comparison

\section{b) $\mathbf{G}_{4}$ based system}

When both the transmitter and the receiver are equipped with 4 antennas, i.e. $N_{r}=N_{t}=4, \mathbf{G}_{4}$ and 16QAM are employed, three turbo codes are of rate $R_{1}=0.94, R_{2}=0.81$ and $R_{3}=0.58$ are used to protect these three data layers. The equivalent coding rate is $R_{c}=(0.94+5 \times 0.81+2 \times 0.58) / 8=0.75$. MLSR coded $\mathbf{G}_{4}$ system employs a single coding rate $R_{c}=0.75$ turbo code, while a coding rate $R_{c}=0.75$ turbo code and QPSK is employed for MLSR coded VBLAST system. All those three schemes are of spectral efficiency of $8 / 4 \times 4 \times 0.75=4 \times 2 \times 0.75=6 \mathrm{bits} / \mathrm{s} / \mathrm{Hz}$. From Fig. 10, when OSIC based detector is used, at BER level of $1 \times 10^{-5}$, MLSR coded $\mathbf{G}_{4}$ system outperforms the MLSR coded VBLAST system 2.2dB. If we employ MLMR coding scheme, further 1.2dB BER performance gain is achieved. In another word, the MLMR coded QO-STBC scheme outperforms the MLSR coded VBLAST scheme $3.2 \mathrm{~dB}$. When ZF detector is employed, the MLMR coded $\mathbf{G}_{4}$ system has BER performance gain up to $7.8 \mathrm{~dB}$. In high SNR region, MLMR coded $\mathbf{G}_{4}$ system with ZF based detector even outperforms the MLSR coded system with OSIC detector. The above simulation results demonstrate that MLMR coded QO-STBC system can achieves significant performance gain over traditional MLSR coded VBLAST system and with acceptable complexity. 


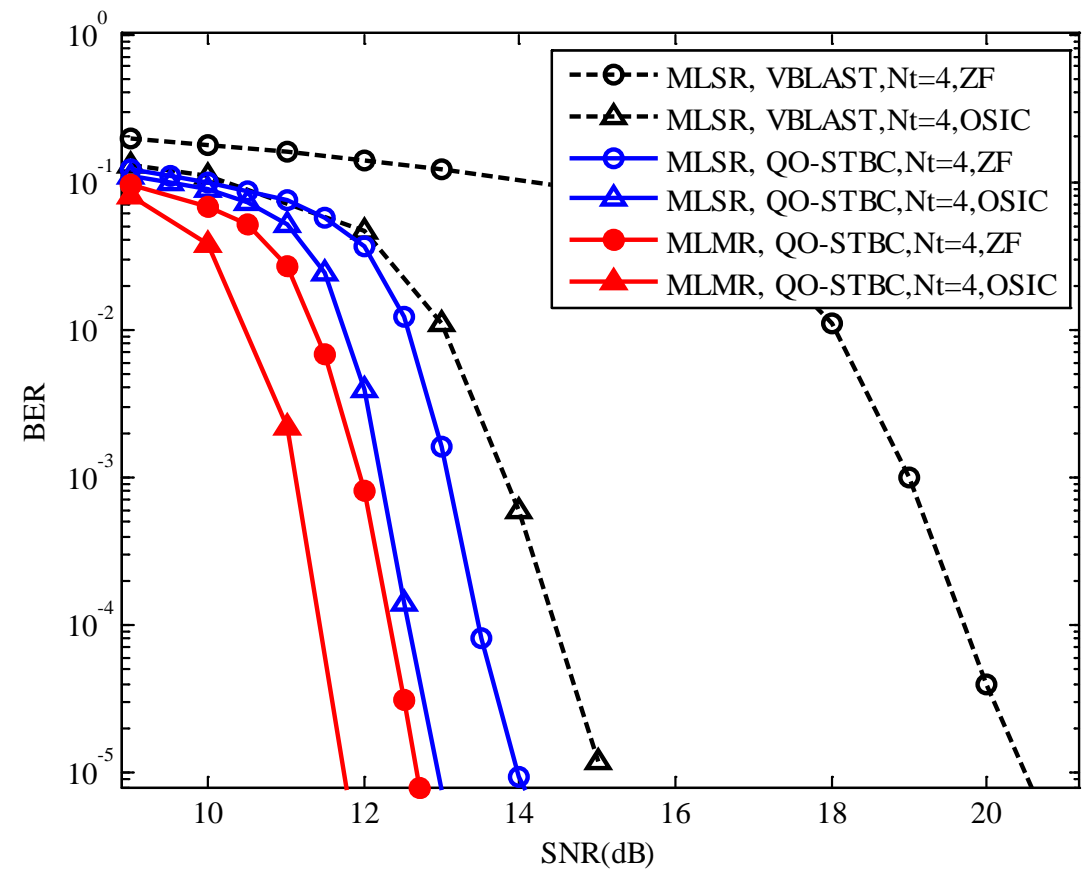

Fig. 10. Under $N_{t}=N_{r}=4$, i.e. $\mathbf{G}_{4}$, BER performance comparison

\subsection{Numerical Link-level throughput simulation result}

Compared with OMA system, the sum-rate performance gain of NOMA system can be defined as $G_{\text {sum }}=\left(C_{\text {sum }}^{\text {NOMA }}-C_{\text {sum }}^{\text {OMA }}\right) / C_{\text {sum }}^{\text {OMA }}$, where $C_{\text {sum }}^{\text {NOMA }}=C_{1}^{(\text {Non-o) }}+C_{2}^{(\text {Non-o })}$ and $C_{\text {sum }}^{\text {OMA }}=C_{1}^{\text {(OMA) }}+C_{2}^{\text {(OMA) }}$. It is reasonable to assume that the bandwidth occupied by each user in OMA system is half of that of users in NOMA system. Thus, for OMA system, the Shannon capacity is $C_{1}^{(\text {OMA })}=0.5 \log _{2}\left(1+S N R_{1}\right)$ for user 1 and $C_{2}^{(\text {OMA })}=0.5 \log _{2}\left(1+S N R_{2}\right)$ for user 2 , the sum-rate gain of user 1 is $G_{1}=\left(C_{1}^{(\text {(Non-o) }}-C_{1}^{(\text {OMA) }}\right) / C_{1}^{(\text {OMA })}$ while it is $G_{2}=\left(C_{2}^{\text {(Non-o) }}-C_{2}{ }^{\text {(OMA) })}\right) / C_{2}{ }^{\text {(OMA) }}$ for user 2. In the simulations, we assume that both the BS and the UEs are equipped with the same number of antennas. EVA channel according to LTE release 10 is employed and CQI period is set to 8ms and the maximum number of HARQ is 4 [23].

In Fig. 11, the theoretic capacity is computed according to the formula above whereas the simulation result is computed according to the link-level throughput. As shown in Fig. 11, when $\mathbf{G}_{3}$ is employed, i.e. $N_{t}=N_{r}=3$ and the SNR gap between these two users is $S N R_{2}-S N R_{1}=15 \mathrm{~dB}$, the performance of the cell-edge user (user 1 ) is improved significantly, while user 2 also gain benefit. Both the theoretic capacity and the simulation results exhibit about the same tendency. At low SNR region, the simulation result is inferior to the theoretic value, whereas it is superior to the theoretic value at middle to high SNR region. This is because we use the CQI and HARQ according to LTE standard OMA system. 


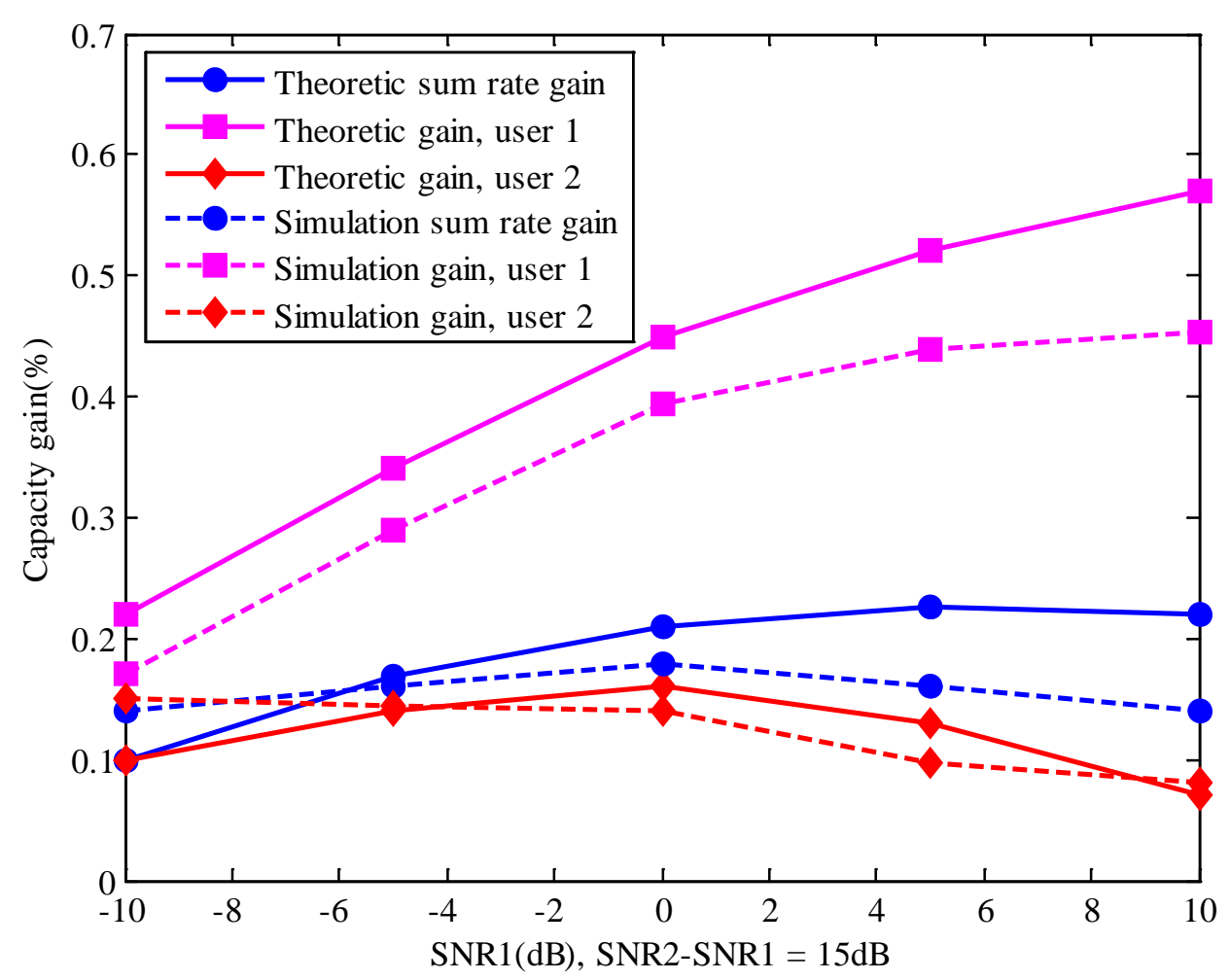

Fig. 11. Link-level throughput gain of NOMA over OMA system when SNR gap is 15dB

\section{Conclusion}

In this paper, we first proposed a P-NOMA scheme joint designed with QO-STBC for MIMO multi-user system. By exploiting the well-designed transmit diversity order, all MIMO data layers have about the same post-detection order, thus the EP of SIC-based detector can be alleviated. Furthermore, we optimized the turbo coding rate of the MLMR-QO-STBC system to further balance the BER of all data layers which leads to improved overall performance. Both BER and link-level throughput performance simulation results show that the proposed joint space-time and power domain NOMA scheme has advantage over the existing P-NOMA and OMA schemes. It is need to point out that the proposed NOMA scheme need not full CSI feedback but the same CSI as LTE standard which make it an attractive scheme for MIMO-NOMA multiuser system. In the future, we intend to design QO-STBC for MIMO-NOMA system with more transmit and receive antennas.

\section{Acknowledgment}

The work of Zeqi Yu was supported by the National Natural Science Foundation of China (Grant no. 61601411), the Science and Technology Project of Henan Province (Grant No. 192102210243) and the Foundation for Young Key Teachers of Zhengzhou University of Light Industry. The works of Jin Xu and Hanqing Ding were supported by the Henan Province important project in Colleges and Universities (Grant no.18B510019). The works of Weihua 
Liu, Zhe Zhang and Xueyan Chen were supported by the National Natural Science Foundation of China (Grant no. 61901418).

\section{Appendix}

1. Power allocation algorithm for two user system

For SIC-based NOMA system, according to Shannon formula, The achievable channel capacity of user 1 can be expressed as

$$
C_{1}^{\text {(Non-o) }}(\alpha)=\log _{2}\left(1+S N R_{1}^{t}\right)=\log _{2}\left(1+\frac{(1-\alpha) G_{1} E_{s_{1}}}{\alpha G_{1} E_{s_{2}}+N_{1}}\right) .
$$

Similarly, the achievable channel capacity of user 2 can be expressed as:

$$
C_{2}^{(\text {Non-o) }}(\alpha)=\log _{2}\left(1+S N R_{2}^{t}\right)=\log _{2}\left(1+\frac{\alpha G_{2} E_{s_{2}}}{N_{2}}\right) .
$$

Where, $\alpha$ denote the power distributin factor, $G_{i}, 1 \leq i \leq 2$ denote the link power gain from BS to user $i, N_{1}=\sigma_{1}^{2}$ and $N_{2}=\sigma_{2}^{2}$. Without loss of generality, we pick up signal on normalized constellation, so $E_{s_{1}}=E_{s_{2}}=1$. To ensure the fairness of the user scheduling, we resort to the weighted sum rate maximization as power allocation criterion.

$$
C_{\text {sum }}=\frac{1}{C_{1}^{(\text {(Non-o) }}(0)} C_{1}^{(\text {Non-o) }}(\alpha)+\frac{1}{C_{2}^{(\text {Non-o) }}(1)} C_{2}^{(\text {Non-o) }}(\alpha) .
$$

In order to solve the optimal power allocation factor $\alpha_{\text {opt }}$, let $\partial C_{\text {sum }} / \partial \alpha=0$, for brevity, we use $C_{1}(0)$ and $C_{2}(1)$ instead of $C_{1}^{(\text {Non-o) }}(0)$ and $C_{2}^{(\text {Non-o) }}(1)$ respectively. We can rewrite formula (A-3) as

$$
C_{\text {sum }}=\frac{1}{C_{1}(0)} C_{1}(\alpha)+\frac{1}{C_{2}(1)} C_{2}(\alpha) .
$$

Then, we need to calculate $\frac{\partial C_{1}(\alpha)}{\partial \alpha}$ and $\frac{\partial C_{2}(\alpha)}{\partial \alpha}$ as follows

(a)

$$
\begin{aligned}
\frac{\partial C_{1}(\alpha)}{\partial \alpha} & =\frac{\partial}{\partial \alpha} \log \left(\frac{G_{1} E_{s_{1}}+N_{1}}{\alpha G_{1} E_{s_{1}}+N_{1}}\right) \\
& =\frac{1}{\ln 2} \frac{\alpha G_{1} E_{s_{1}}+N_{1}}{G_{1} E_{s_{1}}+N_{1}}\left(G_{1} E_{s_{1}}+N_{1}\right)\left(-\frac{G_{1} E_{s_{1}}}{\left(\alpha G_{1} E_{s_{1}}+N_{1}\right)^{2}}\right) \\
& =\frac{-1}{\ln 2} \frac{\alpha G_{1} E_{s_{1}}}{\alpha G_{1} E_{s_{1}}+N_{1}} .
\end{aligned}
$$

(b)

$$
\begin{aligned}
\frac{\partial C_{2}(\alpha)}{\partial \alpha} & =\frac{\partial}{\partial \alpha} \log \left(\frac{\alpha G_{2} E_{s_{2}}+N_{2}}{N_{2}}\right) \\
& =\frac{1}{\ln 2} \frac{N_{2}}{\alpha G_{2} E_{s_{2}}+N_{2}} \frac{1}{N_{2}} G_{2} E_{s_{2}} \\
& =\frac{1}{\ln 2} \frac{G_{2} E_{s_{2}}}{\alpha G_{2} E_{s_{2}}+N_{2}} .
\end{aligned}
$$


Let $\frac{1}{C_{1}(0)} \frac{\partial C_{1}(\alpha)}{\partial \alpha}+\frac{1}{C_{2}(0)} \frac{\partial C_{2}(\alpha)}{\partial \alpha}=0$, we have

$$
\begin{gathered}
\alpha_{o p t}=\frac{\frac{N_{2}}{G_{2} E_{s_{2}}} C_{2}(1)-\frac{N_{1}}{G_{1} E_{s_{1}}} C_{1}(0)}{C_{1}(0)-C_{2}(1)} \\
=\frac{C_{2}(1)-\frac{S N R_{2}}{S N R_{1}} C_{1}(0)}{S N R_{2}\left(C_{1}(0)-C_{2}(1)\right)} .
\end{gathered}
$$

Where $S N R_{1}=G_{1} E_{s_{1}} / N_{1}$ and $S N R_{2}=G_{2} E_{s_{2}} / N_{2}$. According to formula (A-7), the optimal power allocation factor is depend both on the SNR of these two users and the SNR gap between them.

\section{References}

[1] T. Cover, J. Thomas, Elements of Information Theory, John Wiley and Sons, 2005. Article(CrossRefLink).

[2] J. Schaepperle, A. Rüegg, "Enhancement of throughput and fairness in $4 \mathrm{G}$ wireless access systems by non-orthogonal signaling," Bell Labs Technical Journal, vol. 13, no. 4, pp. 59-77, Winter 2009. Article(CrossRefLink).

[3] T. Cover, "Broadcast channel," IEEE Trans. Information Theory, vol. 18, no. 1, pp. 2-14, 1972. Article(CrossRefLink).

[4] S. Tomida and K. Higuchi, "Non-orthogonal access with SIC in cellular downlink for user fairness enhancement," in Proc. of 2011 International Symposium on Intelligent Signal Processing and Communications Systems (ISPACS), Chiang Mai, pp. 1-6, 2011. Article(CrossRefLink).

[5] H. Nikopour and H. Baligh, "Sparse code multiple access," in Proc. of 2013 IEEE 24th Annual International Symposium on Personal, Indoor, and Mobile Radio Communications (PIMRC), London, pp. 332-336, 2013. Article(CrossRefLink).

[6] X. Dai, S. Chen, S. Sun, et al., "Successive interference cancelation amenable multiple access (SAMA) for future wireless communications," in Proc. of 2014 IEEE International Conference on Communication Systems, Macau, pp. 222-226, 2014. Article(CrossRefLink).

[7] 3GPP, R1-162226, "Discussion on multiple access for new radio interface". Article(CrossRefLink).

[8] Li Ping, Lihai Liu, Keying Wu, et al., "Interleave division multiple-access,” IEEE Transactions on Wireless Communications, vol. 5, no. 4, pp. 938-947, April 2006. Article(CrossRefLink).

[9] 3rd Generation Partnership Project, "Technical Specification Group Radio Access Network; Study on Non-orthogonal multiple access (NOMA) for NR,” 3GPP TR 38.812 V0.3.0 Nov. 2018. Article(CrossRefLink).

[10] M. Costa, "Writing on dirty paper (Corresp.)," IEEE Transactions on Information Theory, vol. 29, no. 3, pp. 439-441, May 1983. Article(CrossRefLink).

[11] K. Higuchi, A. Benjebbour, "Non-orthogonal multiple access (NOMA) with successive interference cancellation for future radio access," IEEE Transactions on Communications, vol. E98-B, no. 3, pp. 403-414, Mar. 2015. Article(CrossRefLink).

[12] Z. Ding, M. Peng and H. V. Poor, "Cooperative Non-Orthogonal Multiple Access in 5G Systems," IEEE Communications Letters, vol. 19, no. 8, pp. 1462-1465, Aug. 2015. Article(CrossRefLink).

[13] X. Li, J. Li, Y. Liu, et al., "Outage Performance of Cooperative NOMA Networks with Hardware Impairments," in Proc. of 2018 IEEE Global Communications Conference (GLOBECOM), Abu Dhabi, United Arab Emirates, pp. 1-6, 2018. Article(CrossRefLink).

[14] C. Deng, X. Zhao, D. Zhang, et al., "Performance Analysis of NOMA-based Relaying Networks with Transceiver Hardware Impairments," KSII Transactions on Internet and Information Systems, vol. 12, no. 9, pp. 4295-4316, 2019. Article(CrossRefLink). 
[15] X. Li, J. Li and L. Li, "Performance Analysis of Impaired SWIPT NOMA Relaying Networks Over Imperfect Weibull Channels,” IEEE Systems Journal, pp.1-4, 2019. Article(CrossRefLink).

[16] J. W. Choi, A. C. Singer, J. Lee and N. I. Cho, "Improved linear soft-input soft-output detection via soft feedback successive interference cancellation,” IEEE Transactions on Communications, vol. 58, no. 3, pp. 986-996, March 2010. Article(CrossRefLink).

[17] S. M. Alamouti, “A simple transmit diversity technique for wireless communications," IEEE Journal on Selected Areas in Communications, vol. 16, no. 8, pp. 1451-1458, Oct. 1998. Article(CrossRefLink).

[18] S. Loyka and F. Gagnon, "Performance analysis of the V-BLAST algorithm: an analytical approach,” IEEE Transactions on Wireless Communications, vol. 3, no. 4, pp. 1326-1337, July 2004. Article(CrossRefLink).

[19] X. Dai, "Successive Interference Cancellation Amenable Space-Time Codes with Good Multiplexing-Diversity Tradeoffs,” Wireless personal Communication, vol. 55, pp. 645-654, 2010. Article(CrossRefLink).

[20] J. Xu, W. Ma, X. Dai and Y. Wang, "Joint multi-layer multi-rate code and quasi-orthogonal space-time code scheme with good diversity-multiplexing tradeoffs," in Proc. of 9th International Conference on Communications and Networking in China, Maoming, pp. 266-270, 2014. Article(CrossRefLink).

[21] J. Xu, K. Zhang, “A low-complexity CLSIC-LMMSE-based multiuser detection algorithm for coded MIMO system with high order modulation,” KSII Transaction on Internet and Information System, vol. 11, no. 4, pp. 1954-1971, Apr. 2017. Article(CrossRefLink).

[22] 3rd Generation Partnership Project, “Technical Specification Group Radio Access Network; Evolved Universal Terrestrial Radio Access (EUTRA); Multiplexing and channel coding,” 3GPP TS 36.212 V9.1.0 Mar. 2010. Article(CrossRefLink).

[23] 3rd Generation Partnership Project, “Technical Specification Group Radio Access Network; Evolved Universal Terrestrial Radio Access (EUTRA); Physical layer procedures,” 3GPP TS 36.213 V10.9.0 Feb. 2013. Article(CrossRefLink).

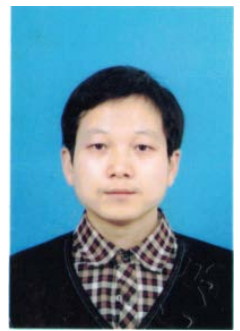

Jin Xu received the B.E. degree in Electronics and Information Engineering from Zhengzhou University in 2005, the M.S. degree in communication and information system from Wuhan University of Technology in 2011, and the Ph.D. degree in Beijing University of Posts and Telecommunications, Beijing, China in 2015, respectively. He is currently a lecturer at School of Computer and Communication Engineering, Zhengzhou University of Light Industry, Zhengzhou. His research interests include LDPC code, iterative decoding algorithm, MIMO detection, and non-orthogonal multiple access.

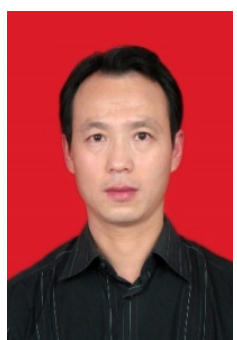

Hanqing Ding received the B.E. degree in information and communication engineering and the Ph.D. degree in military communication from Xidian University, Xian, China, in 2001 and 2011, respectively. He is currently an associate professor at School of Computer and Communication Engineering, Zhengzhou University of Light Industry, Zhengzhou, china. His research interests include wireless communications, resource allocation, and cognitive radio. 

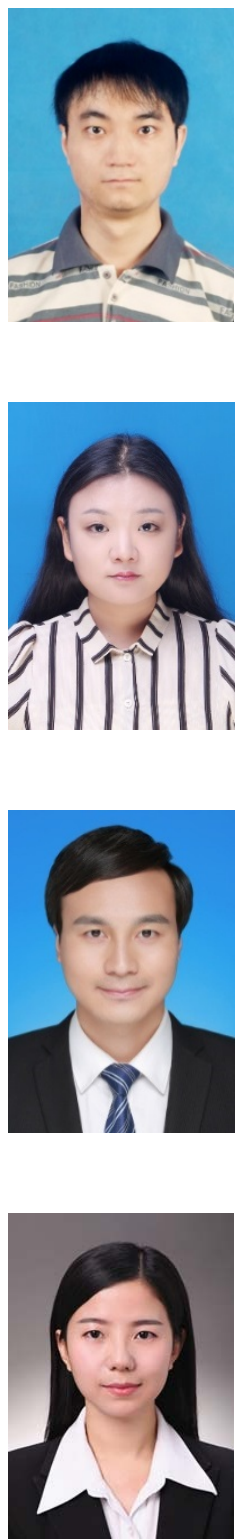

Zeqi Yu received the B.S. degree in Automation from Zhongyuan University of Technology in 2007, the M.S. degree in Control Theory and Control Engineering from Henan University of Technology in 2010, and the Ph.D. degree in information and communication engineering from Northwestern Polytechnical University in 2015, respectively. He is currently a lecturer at School of Computer and Communication Engineering, Zhengzhou University of Light Industry, Zhengzhou. His research interests include digital signal processing and digital IC design.

Zhe Zhang received the B.E. degree in electronic and information engineering from The First Aviation Academy of Chinese Air Force, Xinyang, China, in 2009, and the Ph.D. degree in information and communication engineering from Zhengzhou University, Zhengzhou, in 2017. She is currently a lecturer at School of Computer and Communication Engineering, Zhengzhou University of Light Industry, Zhengzhou. Her research interests include radio resource management and signal processing for wireless communications.

Weihua Liu received the M.S. degree in applied mathematics from Zhengzhou University, Zhengzhou, China, in 2014 and the Ph.D. degree in communication and information system from University of Chinese Academy of Sciences, Beijing, China, in 2018, respectively. He is currently a lecturer at School of Computer and Communication Engineering, Zhengzhou University of Light Industry, Zhengzhou. His research interests include information theory, wireless communication, interference management.

Xueyan Chen received the B.S. degree in Electronic and Information Engineering from PLA Information Engineering University, in 2009. She then received the M.S. degree from Shenyang Ligong University, and Ph. D. degrees from Beijing University of Posts and Telecommunications (BUPT), in 2012 and 2018, respectively. She is currently a lecturer at School of Computer and Communication Engineering, Zhengzhou University of Light Industry, Zhengzhou, China. Her research interests include cognitive radio, relay systems, physical layer security, and energy harvesting. 